\title{
Efficacy and tolerability of a biomineral formulation for treatment of onychoschizia: a randomized trial
}

This article was published in the following Dove Press journal:

Clinical, Cosmetic and Investigational Dermatology

\section{Adele Sparavigna \\ Beatrice Tenconi \\ Laura La Penna}

DERMING, Clinical Research and Bioengineering Institute, Milan, Italy
Correspondence: Adele Sparavigna

Via Valassina 24, 20159 Milan, Italy

Tel +393664004920

Email adele.sparavigna@derming.com
Purpose: Onychoschizia causes lamellar splitting of the nail plate. It is a common problem seen by dermatologists therefore, an effective treatment is needed. The aim of this study was to evaluate the efficacy and tolerability of a biomineral formulation (Biomineral Unghie) applied topically, and/or as an oral supplement, in subjects with onychoschizia.

Patients and methods: This single center, randomized, parallel-group, open-label study was conducted between March 2017 and June 2017. Fifty non-menopausal females aged 18 years or over with onychoschizia were randomized (1:1:1) into three treatment arms to receive either topical and/or oral biomineral formulation for 3 months. Subjects were randomized using a predefined randomization list. The primary objective was to determine the efficacy of the biomineral formulation.

Results: Forty-eight subjects were included in the clinical assessment of nail hardness with 16 subjects in each treatment arm. After 3 months, fingernail hardness improved across the topical, oral and combined treatment arms compared with baseline: 40\% $(P<0.01$ versus untreated hand), $43 \%$ and $50 \%$ ( $P<0.05$ for both), respectively. Image analysis of the nail was carried out on 33 subjects with longitudinal and/or transversal fingernail grooves. Nail roughness was significantly reduced across all arms after 3 months compared with baseline (topical [ $\mathrm{n}=11]$, $-12 \%$; oral supplement $[\mathrm{n}=10],-18 \%$; combined topical/oral $[\mathrm{n}=12],-15 \%$; all $P<0.05$ ). Subjects considered fingernail resistance, smoothness, glossiness, growth and general nail condition to be improved across all treatment arms. No adverse events were reported.

Conclusion: The biomineral formulation was effective in improving nail condition in subjects with onychoschizia after 3 months.

Keywords: nail fragility, nail plate, lamellar splitting, supplements

\section{Plain language summary}

Onychoschizia causes horizontal splitting of the nail and is a common problem in women. Treatment of onychoschizia can be difficult; however, preventative measures and/or oral supplements can be useful in improving nail strength. In this study, we investigated whether a biomineral formulation (Biomineral Unghie; Meda Pharma S.p.A. a Mylan Company, Monza, Italy), applied topically and/or given as an oral supplement, can improve onychoschizia. To do this, we studied female patients with onychoschizia who either applied the topical formulation to the fingernails of their dominant hand once daily and/or consumed the oral supplement once a day. We report that the biomineral formulation was well tolerated and effective in reinforcing and smoothing fingernails in subjects with onychoschizia after 3 months of treatment. Use of either a topical and/or oral biomineral formulation can therefore improve nail condition in patients with onychoschizia. 


\section{Introduction}

Onychoschizia is more commonly known as nail splitting and includes brittle, soft or thin nails. ${ }^{1,2}$ Onychoschizia is a type of nail fragility and is a condition that causes lamellar (horizontal) splitting of the nail plate; ${ }^{3,4}$ it is a common problem seen by dermatologists and occurs at least twice as often in women than men. ${ }^{1,2}$

Although little information is available about the cause of onychoschizia, it is commonly the result of repeated trauma, such as excessive immersion in water with detergents, or the recurrent application of nail polish. ${ }^{5}$ In addition, the frequent use of solvents to remove nail polish can further dehydrate the nail. ${ }^{5}$ One study examined the in vitro nail changes produced by acidic and basic solutions, detergents, water, organic solvents, and other polar materials. It was found that nail clippings exposed to water with alternate periods of drying developed the most prominent lamellar separation. Basic solutions caused some softening, but lamellar separation was only observed after repeated hydration and dehydration. ${ }^{1}$

Other factors associated with onychoschizia can include old age, systemic diseases, drugs and nutritional deficiencies. ${ }^{1,6}$ For example, onychoschizia has been reported to be associated with polycythemia, ${ }^{7,8}$ and ibrutinib treatment for chronic lymphocytic leukemia has been linked to onychoschizia. ${ }^{9,10}$ Dietary deficiencies of certain vitamins and minerals, including iron, zinc and selenium can cause nail alterations leading to onychoschizia. ${ }^{11}$ This suggests that the correct intake of vitamins and minerals is essential for normal nail growth and function.

Treatment of onychoschizia can be difficult, however preventative measures and/or oral supplements can be useful in improving nail strength. ${ }^{6}$ It has been proposed that onychoschizia is associated with chronic wetting and drying, therefore a suggested management approach may involve hydrating the nail followed by an occlusive topical agent that promotes water retention. ${ }^{1}$ Preventative measures can also include wearing gloves whilst performing chores that involve getting the hands wet, avoiding regular application of nail polish, or applying nail polishes that contain nylon fibers that may help strengthen soft nails. ${ }^{2,3,5}$ Excessive manipulation of the nail should also be avoided, although shaping and filing the nail can help prevent further breakage or splitting. ${ }^{2,5}$ The vitamin biotin can be used as an oral supplement and has been tested in subjects with onychoschizia. ${ }^{12}$ A review of clinical trials showed that an improvement in the firmness, hardness, and thickness of brittle nails was seen in subjects treated with oral biotin. ${ }^{13}$ A further trial showed that $91 \%$ of subjects had a definite improvement in fingernail hardness after daily biotin treatment for an average of 5.5 months. ${ }^{14}$ However, larger clinical trials are needed to determine the efficacy and optimal dosing.

This single-center, parallel-group, randomized, open-label study evaluated the efficacy and tolerability of a biomineral formulation (Biomineral Unghie; Meda Pharma S.p.A. a Mylan Company, Monza, Italy) applied topically, and/or as an oral supplement, in subjects with onychoschizia. The topical biomineral formulation is a water and oil based emulsion containing: cystine to harden the nails; a waterresistant oily external phase and panthenol to improve nail resistance; and vitamin E, wheat germ oil and essential lipids to moisturize. The biomineral oral supplement contains amino acids (L-cystine, L-arginine, glutamic acid), vitamins (C, E, B6 and B7 [biotin]), and minerals (zinc, iron and copper) to provide nutrients needed to improve nail condition.

\section{Materials and methods}

\section{Participants}

The study aimed to enroll a total of 50 non-menopausal females aged 18 years or over with onychoschizia. Target enrollment of 50 subjects was intended to obtain at least 48 evaluable cases (16 in each study arm), which was considered by the ethics committee to be sufficient for statistical analysis and to draw general conclusions about the efficacy and tolerability of the biomineral formulation. Subjects were excluded from the study if they were pregnant or lactating, or had other dermatologic conditions affecting the test area (eg, eczema, psoriasis, lesions/scars). Subjects with diabetes; cancer; or endocrine, hepatic, renal, cardiac, pulmonary, neurological, inflammatory or immunosuppressive diseases/ disorders were also excluded. For the duration of the study, subjects were required to avoid changes to their dietary habits, or to detergents or household cleaning products likely to come into contact with hands/nails, and were not permitted to apply cosmetic nail varnish.

\section{Study design and objectives}

This single center, parallel-group, randomized, openlabel study was conducted between March 2017 and June 2017 at the Clinical Research and Bioengineering Institute, Milan, Italy. Subjects were randomized using a predefined randomization list into three treatment arms 


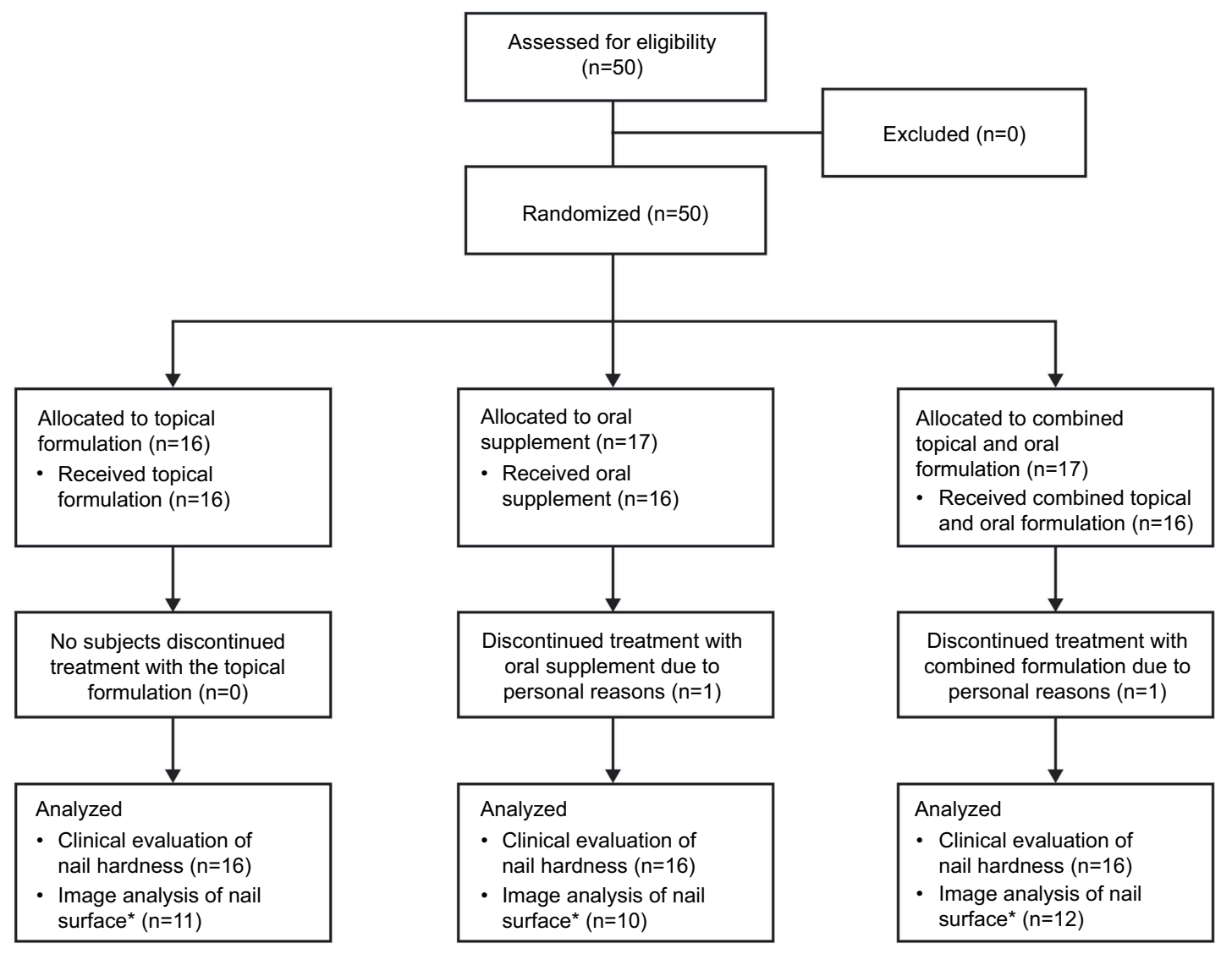

Figure I Subjects enrolled in the study for treatment with topical and/or oral biomineral formulation.

Note: *Image analysis was only carried out on subjects with longitudinal and/or transversal fingernail grooves $(n=33)$.

$(1: 1: 1)$ to receive either topical and/or oral ingestion of the biomineral formulation for 3 consecutive months (Figure 1).

The primary objective of the study was to determine the efficacy of the biomineral formulation applied topically and/or as an oral supplement. The secondary objectives were to evaluate the tolerability of each formulation assessed by the investigator and the subjects.

This study was conducted in accordance with the principles of the Declaration of Helsinki and ethical approval was granted by the DERMING independent ethical committee on February 17, 2017. All subjects provided written informed consent.

\section{Treatment}

The topical formulation was applied to the fingernails of the dominant hand once daily. Before each application, the subjects washed their hands with soap and water and dried them thoroughly. Fingernails of the non-dominant hand were used as controls so nail condition could be compared within each subject. The oral formulation was in the form of a food supplement capsule and was consumed once a day with a glass of water during a meal. Subjects who were randomized into the third treatment arm applied both the topical formulation and consumed the oral supplement each day.

\section{Outcomes}

Efficacy parameters were assessed at baseline, and after 1 and 3 months of treatment. Fingernail hardness was assessed by applying constant pressure to the nail of the third finger on the treated and untreated hands. A clinical score was assigned according to a scale whereby $0=\mathrm{soft}$ nails, 1=slightly hard nails, 2=moderately hard nails, $3=$ very hard nails. All assessments were performed by the same investigator in order to reduce subjectivity and variability. Fingernail roughness was evaluated by noninvasive 3D profilometry using a Primos compact portable device (GFMesstechnik GmbH), which generated 3D representations of the nail surface and was equipped with software to calculate an average value for nail roughness 
(Ra value $[\mu \mathrm{m}])$. At each time point, a picture of the nail surface was taken using a digital camera; patients provided written informed consent for the production of photographic images. At the end of the trial, each subject assessed the efficacy and tolerability of both the topical and oral biomineral formulations by completing a questionnaire.

\section{Statistical analysis}

Clinical data were analyzed using the Friedman test to compare different timepoints versus baseline. If a statistically significant result was yielded, a Holm-Sidak Adjusted Wilcoxon signed rank test was performed.

Instrumental data were analyzed with: 1) a non-parametric test (Friedman Test), if the null hypothesis was rejected by Shapiro-Wilk normality test (threshold at 5\%); and 2) a parametric test (analysis of variance [ANOVA] test for repeated measures), if the null hypothesis was confirmed by Shapiro-Wilk normality test (threshold at 5\%). In case of statistically significant results Holm-Sidak Adjusted tests were performed.

The efficacy of the topical formulation and/or the oral supplement was expressed in absolute values versus baseline (T0) and in comparison with each other. For the topical formulation, the efficacy was also expressed versus the untreated hand (comparison within subjects).

\section{Results}

A total of 50 subjects enrolled in the study, however, two drop-outs occurred (one subject from the oral supplement treatment arm and one subject from the combined topical and oral treatment arm [Figure 1]). Drop-outs were due to personal reasons rather than the products being tested. The 48 remaining patients were aged $18-54$ years, with mean ages of 39-45 years across treatment groups (Table 1).
Forty-eight subjects were included in the clinical assessment of nail hardness; there were 16 subjects in each study arm, with mean baseline scores for nail hardness ranging from 1.0 to 1.4 (Table 1). Image analysis of the nail was only carried out on subjects with longitudinal and/or transversal fingernail grooves $(n=33)$ : 11 subjects were included in the topical formulation treatment arm, 10 subjects were in the oral supplement arm and 12 subjects were in both the topical and oral treatment arm.

\section{Clinical evaluation of nail hardness}

Improvements in fingernail hardness were observed across all treatment arms (Figure 2). In the topical formulation arm, nail hardness increased by $40 \%$ after 3 months of treatment compared with baseline $(P<0.01$ versus the untreated hand). In subjects who consumed the oral supplement, nail hardness increased by $43 \%$ compared with baseline after 3 months of treatment $(P<0.05)$. Subjects who applied the topical formulation and ingested the oral supplement had an improvement in nail hardness of $50 \%$ compared with baseline $(P<0.05)$. After 3 months of treatment, nail hardness on the untreated hand was reduced by $-10 \%$ compared with baseline. Figure 3 shows improvements in lamellar splitting after application of the topical and/or oral biomineral formulation, after 1 and 3 months of treatment.

\section{Image analysis of nail surface}

Image analysis indicated a significant reduction in nail roughness after 1 month of topical biomineral formulation $(-12 \% ; P<0.05)$ and combined topical and oral treatment $(-8 \% ; P<0.05)$ versus baseline (Figures 4 and 5$)$. Nail roughness was significantly reduced across the topical, oral and combined treatment arms after 3 months of

Table I Baseline demographics and clinical characteristics

\begin{tabular}{|c|c|c|c|}
\hline \multirow[t]{2}{*}{ Characteristics } & \multicolumn{3}{|c|}{ Treatment arms $(\mathrm{N}=48)^{\mathrm{a}}$} \\
\hline & $\begin{array}{l}\text { Ist arm } \\
\text { (topical formulation) } \\
N=16\end{array}$ & $\begin{array}{l}\text { 2nd arm } \\
\text { (oral supplement) } \\
\mathbf{N}=16\end{array}$ & $\begin{array}{l}\text { 3rd arm } \\
\text { (topical formulation and oral supplement) } \\
N=16\end{array}$ \\
\hline Mean age, years & 45 & 42 & 39 \\
\hline Age range, years & $22-53$ & $21-53$ & $18-54$ \\
\hline Mean nail hardness score ${ }^{b}$ & 1.0 & 1.4 & 1.2 \\
\hline
\end{tabular}

Notes: ${ }^{a}$ Fifty subjects enrolled in the study; however, two drop-outs occurred due to personal reasons rather than the products being tested. ${ }^{b}$ Nail hardness scores on $0-3$ scale ( $0=$ soft nails; $I=$ slightly hard nails; $2=$ moderately hard nails; $3=$ very hard nails). 


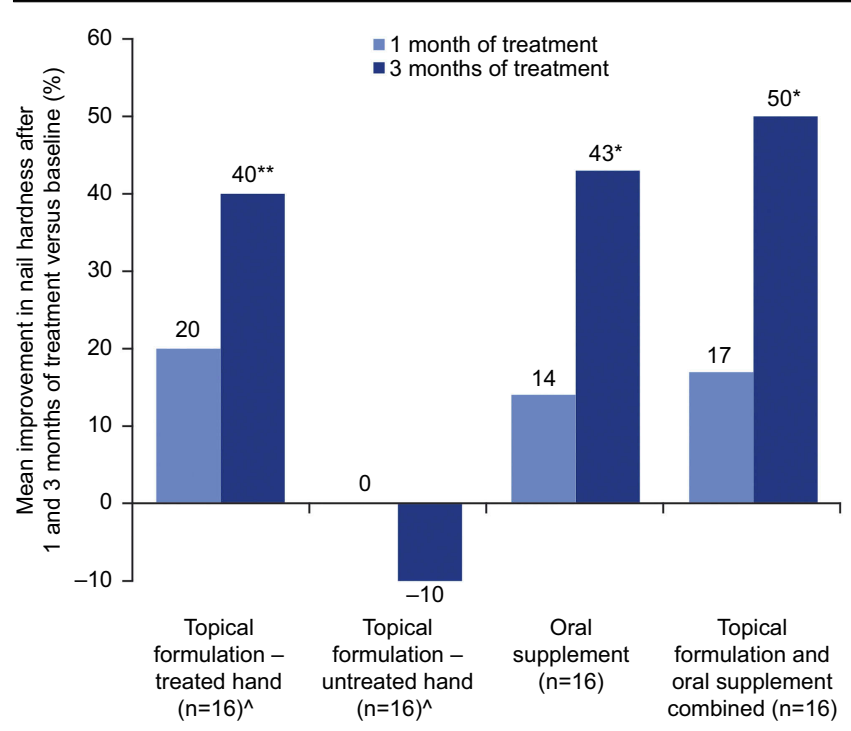

Figure 2 Mean improvements in fingernail hardness after topical and/or oral biomineral formulation treatment.

Notes: *Holm-Sidak Adjusted Wilcoxon signed rank test, $P<0.05$ versus baseline; **Wilcoxon signed rank test, $P<0.0$ I versus untreated hand; ${ }^{\wedge}$ The topical formulation was applied to the fingernails of the dominant hand once daily, whilst fingernails of the non-dominant hand were used as controls, so that nail condition could be compared within each subject. treatment compared with baseline $(-12 \% ;-18 \% ;-15 \%$, respectively; all $P<0.05$ ) (Figures 4 and 5). In contrast, nail roughness on the untreated hand was increased by $9 \%$ versus baseline, following both 1 month and 3 months of treatment.

\section{Self-evaluation questionnaire}

The majority of subjects considered fingernail resistance, smoothness, glossiness, growth and general nail condition to be improved across all treatment arms (Table 2).

\section{Tolerance and safety}

$100 \%$ of subjects rated the tolerance of both the topical and oral biomineral formulation as good or excellent, which was further confirmed by the findings of the investigator who, during the trial, did not register any type of adverse event to the topical and/or oral biomineral formulations.

\section{A}

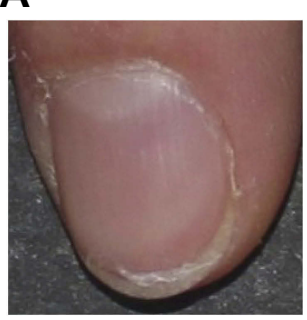

Baseline

\section{B}

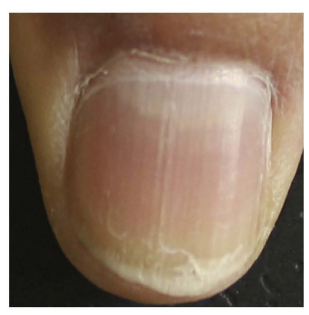

Baseline

C

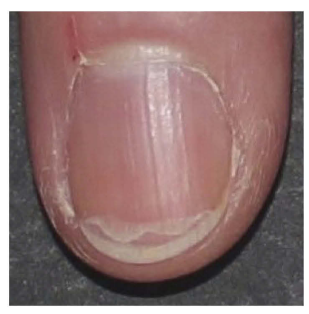

Baseline

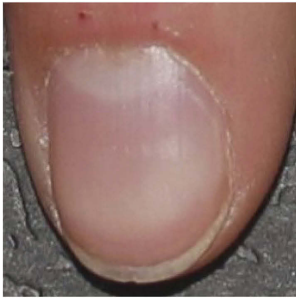

1 month

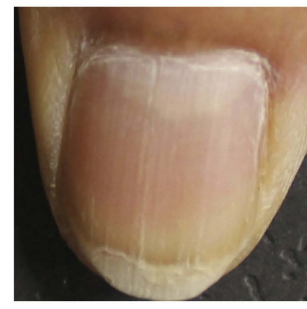

1 month

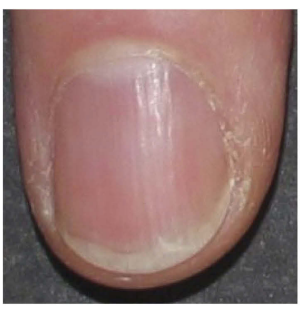

1 month

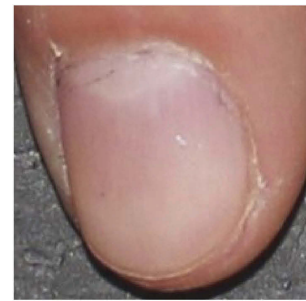

3 months

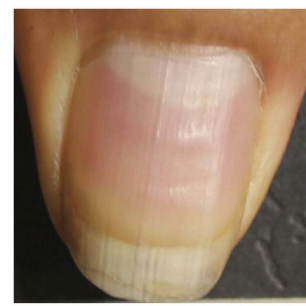

3 months

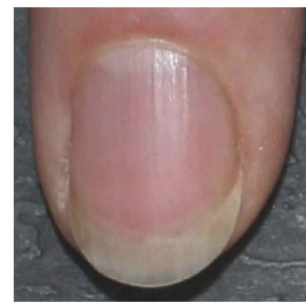

3 months

Figure 3 Clinical pictures showing the effect of the topical and/or oral biomineral formulation on lamellar splitting.

Note: (A) Nail condition after treatment with topical formulation; (B) nail condition after treatment with oral supplement; (C) nail condition after treatment with topical formulation and oral supplement combined. 


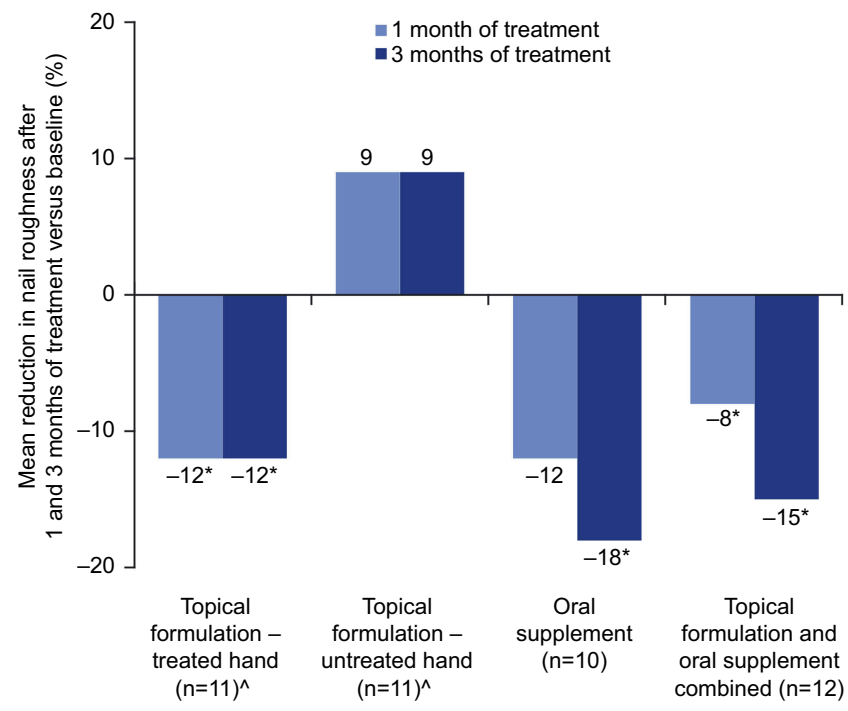

Figure 4 Mean reduction in nail roughness after topical and/or oral biomineral formulation treatment.

Note: *Holm-Sidak Adjusted Paired $t$-test, $P<0.05$ versus baseline; $\wedge^{\text {the }}$ topical formulation was applied to the fingernails of the dominant hand once-daily, whilst fingernails of the non-dominant hand were used as controls, so that nail condition could be compared within each subject.

\section{Discussion}

In this randomized trial, it was observed that the biomineral formulation, when applied topically and/or used as an oral supplement, reinforced and smoothed fingernails in women with onychoschizia.

The greatest improvement in nail hardness was seen after 3 months of treatment with the combined topical and oral biomineral formulation compared with baseline $(50 \% ; P<0.05)$ whereas, the greatest reduction in nail roughness was seen after 3 months of treatment with the oral supplement versus baseline $(-18 \% ; P<0.05)$. No significant increase in nail hardness was seen with either the topical and/or oral biomineral formulation after 1 month of treatment, however, nail hardness was significantly increased across all treatment arms after 3 months compared with baseline. In comparison, there was a significant reduction in nail roughness after 1 month of treatment in both the topical and combined biomineral formulation arms compared with baseline. A

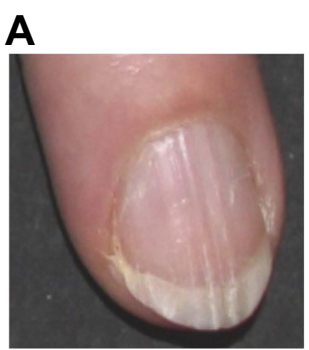

Baseline

B

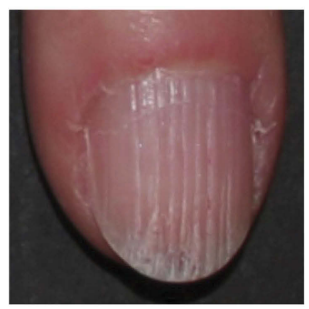

Baseline

C

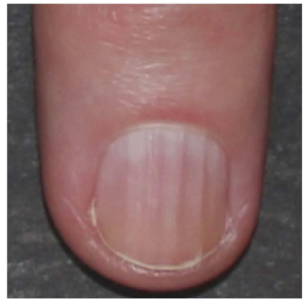

Baseline

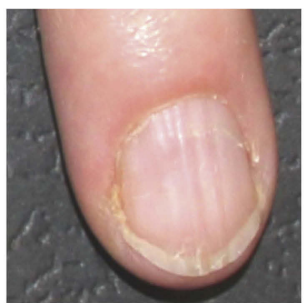

1 month

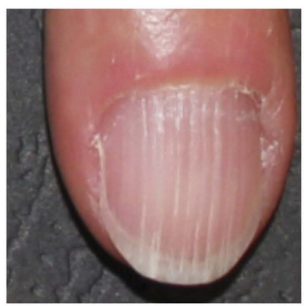

1 month

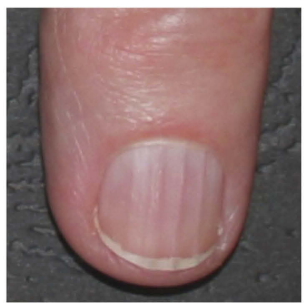

1 month

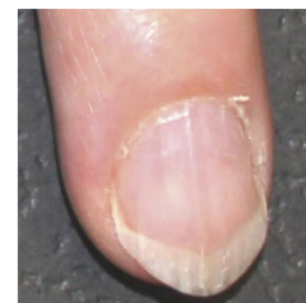

3 months

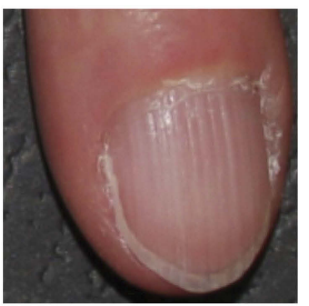

3 months

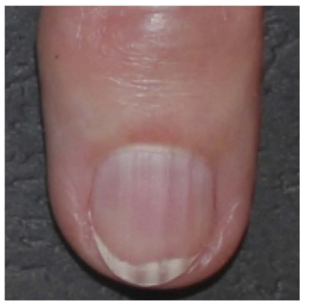

3 months

Figure 5 Clinical pictures showing the effect of the topical and/or oral biomineral formulation on nail roughness.

Note: (A) Nail condition after treatment with topical formulation; (B) nail condition after treatment with oral supplement; (C) nail condition after treatment with topical formulation and oral supplement combined. 
Table 2 Subject assessment of the efficacy of topical and/or oral biomineral formulation

\begin{tabular}{|c|c|c|c|}
\hline & \multicolumn{3}{|c|}{$\begin{array}{l}\text { Percentage of subjects with medium, marked and very marked improvement in nail } \\
\text { condition }\end{array}$} \\
\hline & $\begin{array}{l}\text { Ist arm } \\
\text { (topical formulation) } \\
N=16\end{array}$ & $\begin{array}{l}\text { 2nd arm } \\
\text { (oral supplement) } \\
\mathrm{N}=16\end{array}$ & $\begin{array}{l}\text { 3rd arm } \\
\text { (topical formulation and oral supplement) } \\
N=16\end{array}$ \\
\hline Nail resistance (\%) & 75 & 88 & 81 \\
\hline Nail smoothness (\%) & 75 & 82 & 75 \\
\hline Nail glossiness (\%) & 75 & 75 & 81 \\
\hline Nail growth speed (\%) & 63 & 88 & 62 \\
\hline General nail condition (\%) & 63 & 75 & 75 \\
\hline
\end{tabular}

significant reduction in nail roughness was observed across all three treatment arms after 3 months versus baseline.

Other studies have also shown improvements in nail condition with both topical and oral formulations. For example, in one study, 60 subjects were randomized and applied $10 \%$ urea lacquer once- $(n=30)$ or twice- $(n=30)$ daily on one hand for 28 days using the other hand as a control. The study demonstrated significant improvements in nail surface morphology and nail plate consistency with the urea lacquer. ${ }^{15}$ In addition, daily ingestion of bioactive collagen peptides promoted increases in nail growth and reduced the frequency of cracked and/or chipped nails in subjects with brittle nails. ${ }^{16}$

Although the majority of subjects in this study considered fingernail resistance, smoothness, glossiness, growth and general nail condition to be improved across all treatment arms, it can be seen that subjects rated the oral supplement higher than the topical and combined biomineral formulations in nail resistance, nail smoothness, and growth speed. The efficacy of treatment options may have been further improved with a longer course of treatment and/or a longer follow-up period, as demonstrated in a study from Switzerland where patients experienced a $25 \%$ increase in nail plate thickness after receiving biotin over a 6 month period. ${ }^{17}$

\section{Limitations}

A potential limitation of this study was that it was only carried out in females therefore, results cannot be generalized. However, as women are affected by onychoschizia twice as frequently as men, ${ }^{18}$ and the intercellular keratinocyte bridges are thought to be constitutionally weaker in women than in men, ${ }^{6}$ this inclusion criterion was deemed acceptable for this study. An additional limitation may have been the open label study design, which could have introduced the potential for bias through unblinding. Furthermore, clinical scoring of nail hardness was a subjective measure, although all assessments were performed by the same investigator to minimize variation. Finally, only 33 subjects were included in the image analysis of the nail, whereas 48 subjects were included in the clinical assessment of nail hardness. Although the Ethics committee considered the sample sizes of the study to be sufficient to draw general conclusions about the efficacy and tolerability of the biomineral formulation, both sample sizes are still relatively small.

\section{Conclusion}

In conclusion, treatment with topical and/or oral biomineral formulation was effective in reinforcing and smoothing fingernails in subjects with onychoschizia after 3 months of treatment. Treatment was also well tolerated. These findings provide evidence to support the use of either the topical and/or oral biomineral formulation in the treatment of onychoschizia.

\section{Ethics approval and informed consent}

This study was conducted in accordance with the principles of the Declaration of Helsinki and ethical approval was granted by the DERMING independent ethical committee on February 17, 2017. All subjects provided written informed consent.

\section{Data availability}

The datasets generated during and/or analyzed during the current study are available from the corresponding author on reasonable request. 


\section{Acknowledgment}

Medical writing assistance in the preparation of this manuscript was provided by Laura Brennan (CircleScience, an Ashfield Company, part of UDG Healthcare plc), and funded by Meda Pharma S.p.A., a Mylan company.

\section{Author contributions}

All authors contributed to data analysis, drafting and revising the article, gave final approval of the version to be published, and agree to be accountable for all aspects of the work.

\section{Disclosure}

DERMING, Clinical Research and Bioengineering Institute has received research fees from Meda Pharma $\mathrm{S}$. p.A., a Mylan company. The authors report no other conflicts of interest in this work.

\section{References}

1. Wallis MS, Bowen WR, Guin JD. Pathogenesis of onychoschizia (lamellar dystrophy). J Am Acad Dermatol. 1991;24(1):44-48. doi:10.1016/0190-9622(91)70007-O

2. AOCD. Dermatologic Disease Database: Brittle Splitting Nails. 2018. Available from: http://www.aocd.org/?page=BrittleSplit tingNail. Accessed October 5, 2018

3. Skinsight. Skin conditions: Nail Splitting (Onychoschizia). Information for adults. 2018. Available from: http://www.skinsight.com/skin-condi tions/adult/onychoschizia?Imiw9cApl. Accessed October 5, 2018

4. Baran R, Schoon D. Nail fragility syndrome and its treatment. $J$ Cosmet Dermatol. 2004;3(3):131-137. doi:10.1111/j.1473-2130.2004.00076.x
5. Paller A, Mancini AJ, Hurwitz S. Hurwitz Clinical Pediatric Dermatology: A Textbook of Skin Disorders of Childhood and Adolescence. 5th ed. New York: Elsevier/Saunders; 2011.

6. Iorizzo M, Pazzaglia M, Piraccini BM, Tullo S, Tosti A. Brittle nails. $J$ Cosmet Dermatol. 2004;3(3):138-144. doi:10.1111/j.1473-2130.2004. 00084.x

7. Keohane C, McMullin MF, Harrison C. The diagnosis and management of erythrocytosis. BMJ. 2013;347:f6667. doi:10.1136/bmj.f6667

8. Motswaledi M, Mayayise M. Nail changes in systemic diseases. SAAFP. 2010;52(5):409-413. doi:10.1080/20786204.2010.10874016

9. Heldt Manica LA, Cohen PR. Ibrutinib-associated nail plate abnormalities: case reports and review. Drug Saf Case Rep. 2017;4(1):15. doi:10.1007/s40800-017-0060-1

10. Bitar C, Farooqui MZ, Valdez J, et al. Hair and nail changes during long-term therapy with ibrutinib for chronic lymphocytic leukemia. JAMA Dermatol. 2016;152(6):698-701. doi:10.1001/jamader matol.2016.0225

11. Cashman MW, Sloan SB. Nutrition and nail disease. Clin Dermatol. 2010;28(4):420-425. doi:10.1016/j.clindermatol.2010.03.037

12. Colombo VE, Gerber F, Bronhofer M, Floersheim GL. Treatment of brittle fingernails and onychoschizia with biotin: scanning electron microscopy. J Am Acad Dermatol. 1990;23(6 Pt 1):1127-1132. doi:10.1016/0190-9622(90)70345-I

13. Lipner SR, Scher RK. Biotin for the treatment of nail disease: what is the evidence? J Dermatolog Treat. 2018;29(4):411-414. doi:10.1080/ 09546634.2017.1395799

14. Floersheim GL. [Treatment of brittle fingernails with biotin]. $Z$ Hautkr. 1989;64(1):41-48.

15. Kruger N, Reuther T, Williams S, Kerscher M. [Effect of urea nail lacquer on nail quality. Clinical evaluation and biophysical measurements]. Hautarzt. 2006;57(12):1089-1094. doi:10.1007/s00105-006-1215-7

16. Hexsel D, Zague V, Schunck M, Siega C, Camozzato FO, Oesser S. Oral supplementation with specific bioactive collagen peptides improves nail growth and reduces symptoms of brittle nails. $J$ Cosmet Dermatol. 2017;16(4):520-526. doi:10.1111/jocd.12393

17. Hochman LG, Scher RK, Meyerson MS. Brittle nails: response to daily biotin supplementation. Cutis. 1993;51(4):303-305.

18. van de Kerkhof PC, Pasch MC, Scher RK, et al. Brittle nail syndrome: a pathogenesis-based approach with a proposed grading system. $\mathrm{J} \mathrm{Am}$ Acad Dermatol. 2005;53(4):644-651. doi:10.1016/j.jaad.2004.09.002

\section{Publish your work in this journal}

Clinical, Cosmetic and Investigational Dermatology is an international, peer-reviewed, open access, online journal that focuses on the latest clinical and experimental research in all aspects of skin disease and cosmetic interventions. This journal is indexed on CAS.
The manuscript management system is completely online and includes a very quick and fair peer-review system, which is all easy to use. Visit http://www.dovepress.com/testimonials.php to read real quotes from published authors. 